\title{
EECP Sistem Uygulamasında ST Segmentinin Yapay Sinir Ağları ile Belirlenmesi *
}

\author{
Hanife Satoğlu Hasbenlioğlu ${ }^{1 \dagger}$, Kemal Kaya ${ }^{2}$, Kenan Danışman ${ }^{2}$ \\ ${ }^{1}$ Erciyes Üniversitesi, Fen Bilimleri Fakültesi, Elektrik Elektronik Bölümü, Kayseri, Türkiye (ORCID: 0000-0000-0000-0000) \\ ${ }^{2}$ Erciyes Üniversitesi, Klinik Mühendisliği Uygulama ve Araştırma Merkezi, Kayseri, Türkiye (ORCID: 0000-0002-1254-422X)
}

(Konferans Tarihi: 5-7 Mart 2020)

(DOI: $10.31590 /$ ejosat.araconf56)

ATIF/REFERENCE: Satoğlu Hasbenlioğlu, H., Kaya, K. \& Danı̧̧man, K. (2020). EECP Sistem Uygulamasında St Segmentinin Yapay Sinir Ağlari ile Belirlenmesi. Avrupa Bilim ve Teknoloji Dergisi, (Özel Say1), 430-435.

$\ddot{O} \mathbf{z}$

Bu çalışmada gelişmiş harici karşı darbe (External Enhanced Counter Pulsation-EECP) cihazının işaret işleme biriminin kardiyovasküler dolaşımın diyastol faz başlangıcını hatasız biçimde tespit edebilmesini sağlamak için elektrokardiyogram (EKG) işaretinin ST parçasının segmentasyonu gerçekleştirilmiştir. EECP cihazı özellikle miyokard iskemisinin non-invazif tedavisinde kullanılmaktadır. Cihaz (EKG) işaretini analiz etmekte ve kan dolaşımının diyastol fazında hastanın alt uzuvlarına bağlı manşonları şişirerek haricen basınç uygulamaktadır. Dolaşımın sistol fazında ise manşonlar söndürülerek harici basıncın ortadan kaldırılması suretiyle sistemik vasküler rezistansın azalması sağlanmaktadır. Bu etki kalbin art yükünün (afterload) düşmesine yol açar. EKG işaretinin QRS parçası ventrikülün (karıncık) kasılmasını başlatır. Bir başka ifadeyle QRS dalgasının ortaya çıkışından hemen sonra kalp kasılması yani sistol fazı başlar. T dalgasının ortaya çıkışııı takiben ventrikülün kasılması son bulur. Dolayısıyla EECP cihazının doğru zamanda devreye girmesini sağlamak için ST dalga kompleksinin tespit edilmesi bir zorunluluktur.

Yapılan çalı̧̧mada ST segmentasyonu için Physio.net'in sağlamış olduğu MIT-BIH Arrhythmia Database kullanılmıştır. Sisteme yüklenen işaretler öncesinde ön işleme tabi tutularak gürültülerden ve baseline kaymalarından arındırılmıştır. Gürültü eliminasyonu ve baseline kayması düzenlenen işaretin $\mathrm{P}, \mathrm{Q}, \mathrm{R}, \mathrm{S}$ ve T noktaları tespit edilmiştir. Tespit edilen bu noktalardan $\mathrm{P}, \mathrm{Q}$ ve $\mathrm{R}$ noktalarıyla birlikte bu noktalarla ilişkili 7 nokta daha giriş işaretlerini oluşturarak 10 girişli ve S, T noktalarını belirleyecek şekilde 2 çıkışlı bir ara katmanlı bir yapay sinir ağı oluşturulmuştur. Oluşturulan bu yapıda k cross validation (k katlamalı çaprazlama) yöntemi kullanılmıştır. Sistem eğitiminde sabit eğitim algoritması, farklı k katsayıları, farklı nöron sayıları ve farklı transfer fonksiyonları kullanılarak en iyi yapı elde edilmeye çalışılmıştır. Sabit olarak seçilen eğitim algoritması Levenberg-Marquardt’dır. Yapılan çalışma sonucunda k katlama değeri 5 olarak belirlenmiştir. 25 nöronun kullanıldığı gizli katman için logsig tipi transfer fonksiyonu ve çıkış için poslin tipi transfer fonksiyonu kullanılarak en küçük hatayı veren yapı elde edilmiştir.

Anahtar Kelimeler: EECP, Yapay Sinir Ağları, ST Segmentasyonu

\section{Determination of ST Segment in EECP System Application by Artificial Neural Networks}

\begin{abstract}
In this study in order to detect start of diastole phase of blood cicrculation by signal processing unit of EECP (enhanced exeternal counterpulsation device) device segmentation of ST part of ECG (electrocardiogram) signal has been done. EECP device is especially being used in the non invasive treatment of myocardial ischemia. Device analyses ECG signal and then in the diastole phase applys external pressure to lower extremities by inflating the cuffs with air weared by patient on his/her lower extremities. In the systole
\end{abstract}

\footnotetext{
${ }^{*}$ Bu makale International Conference on Access to Recent Advances in Engineering and Digitalization (ARACONF 2020) de sunulmuştur.

† Sorumlu Yazar: Erciyes Üniversitesi, Fen Bilimleri Fakültesi, Elektrik Elektronik Bölümü, Kayseri, Türkiye, hanife.satoglu@gmail.com
} 
phase systemic vascular resistance decreases when the device deflates the cuffs which removes external pressures on extremities. This also results in reduce of heart's afterload. QRS wave initiates to contraction of heart's ventricles. In other words the systole phase begins shortly after the appearance of QRS wave. The ventricle's contraction is ended following the appearance of T wave. Hence, it is required to detect ST complex of the ECG signal correctly so as to run the EECP at exact time intervals. MIT-BIH Arrhythmia Database provided by Physio.net is used for ST segmentation. Firstly, the signals, which are loaded at system, are subjected to pre processing and purify the baseline shifting. After this operation $\mathrm{P}, \mathrm{Q}, \mathrm{R}, \mathrm{S}$ and $\mathrm{T}$ dots are detected. In the detected dots of $\mathrm{P}, \mathrm{Q}, \mathrm{R}$ and the other 7 relevant dots, which are near to $\mathrm{P}, \mathrm{Q}$ and $\mathrm{R}$ dots, are selected and giving the system as 10 input. The other dots $\mathrm{S}$ and $\mathrm{T}$ are creates the 2 outputs. Current used structure is a neural network artificial and has 10 inputs, 2 outputs and one layer. At this study, it is used to $\mathrm{k}$ cross validation. For system training it is used stabil train algorithm, different neuron number and different transfer functions. The stabil train algorithm is Levenberg-Marquardt. At the end of study folding value $\mathrm{k}$ is determined as 5 . The structure that results in smallest error is acquired through a hidden layer of 25 neurons of which transfer function is in type of logsig and a poslin type transfer function for the output.

Keywords: EECP, Neural Network Artificial, ST Segmentation

\section{Giriş}

Kalp hastalıkları, bulaşıcı olmayan hastalıklar içerisinde Türkiye ve Dünyada ilk sırada yer almakta ve bu hastalık sebebiyle her yıl binlerce kişi hayatını kaybetmektedir. Kalp hastalıklarının teşhisinde kullanılan en önemli tıbbi cihaz EKG cihazıdır. EKG cihazı, hastanın göğsünün farklı yerlerine yerleştirilen problar vasıtasıyla kalbin elektriksel aktivitesini ölçüp kaydeden, oldukça düşük maliyetli non-invasive bir cihazdır. EKG sinyali, band genişliği $0,05 \mathrm{~Hz}$ ile $100 \mathrm{~Hz}$ arasında değişen tipik olarak tepeden tepeye genlik değeri $5 \mathrm{mV}$ 'olan bir AC sinyaldir [1]. EKG işareti, Şekil 1'de görüldüğü gibi P, Q, R, S ve T noktalarının belirlediği tepe ve çukurlardan oluşmaktadır. Hastalık teşhisinde bu noktaların konumlarına ve durumlarına bakılarak yorum yapılıp tedavi yöntemleri geliştirilmektedir.

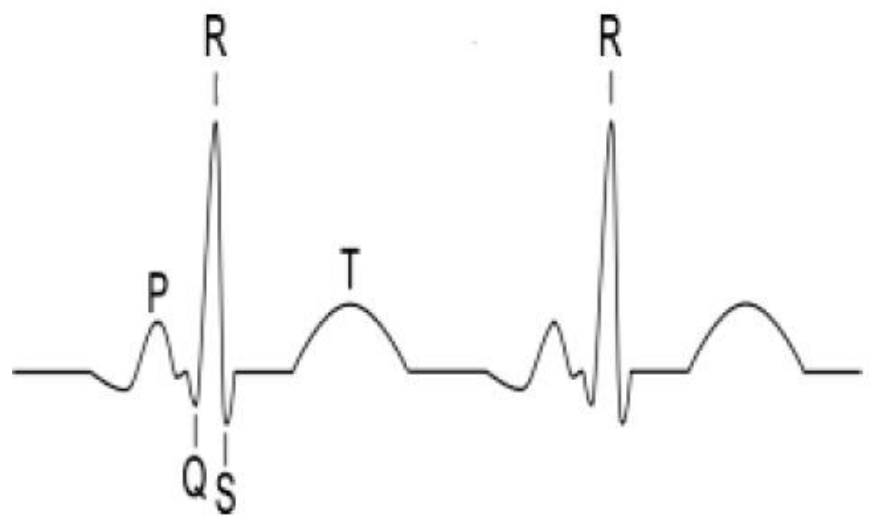

Şekil 1: EKG İşareti

EECP'de bu süreçte geliştirilmiş olan EKG tabanlı non-invasive bir yöntemdir. Bu yöntem 1950'li yılların başlarında Amerika Birleşik Devletleri'nde kullanılmaya başlanmıştır. EECP'nin ilk kullanım amacı miyokard iskemisi olan hastalarda miyokardın oksijen ihtiyaç miktarını ayarlamayı amaçlamıştır. İlerleyen zamanlarda bu tedavi yöntemi kalp krizi ve inme geçiren hastalarda da kullanılmaya başlanmıştır. Günümüzde diğer kalp hastalıkları için de EECP yöntemi kullanılarak tedavide ne kadar başarılı olunduğu araştırılmaktadır [2, 3, 4].

EECP yöntemi, Şekil 2'de gösterildiği gibi bacaklara bağlanan manşonların sırasıyla sıkılıp gevşetilmesi ilkesine göre çalışmaktadır. Vücudun kalp haricindeki tüm organ ve dokuları sistol fazında kanlanırken kalbin kendisi diyastol fazında kanlanır. Sistol fazında cihaz manşon basıncını azaltarak sistemik vasküler rezistansın azalmasını sağlar. Bu etki ise kalbin art yükünün (afterload) düşmesine yol açar. QRS dalgası ventrükülün kasılmasını başlatır. Bir başka ifadeyle QRS dalgasının ortaya çıkışından hemen sonra sistol başlar. T dalgasının ortaya çıkışını takiben ventrikülün kasılması son bulur ve manşonlar gevşer.

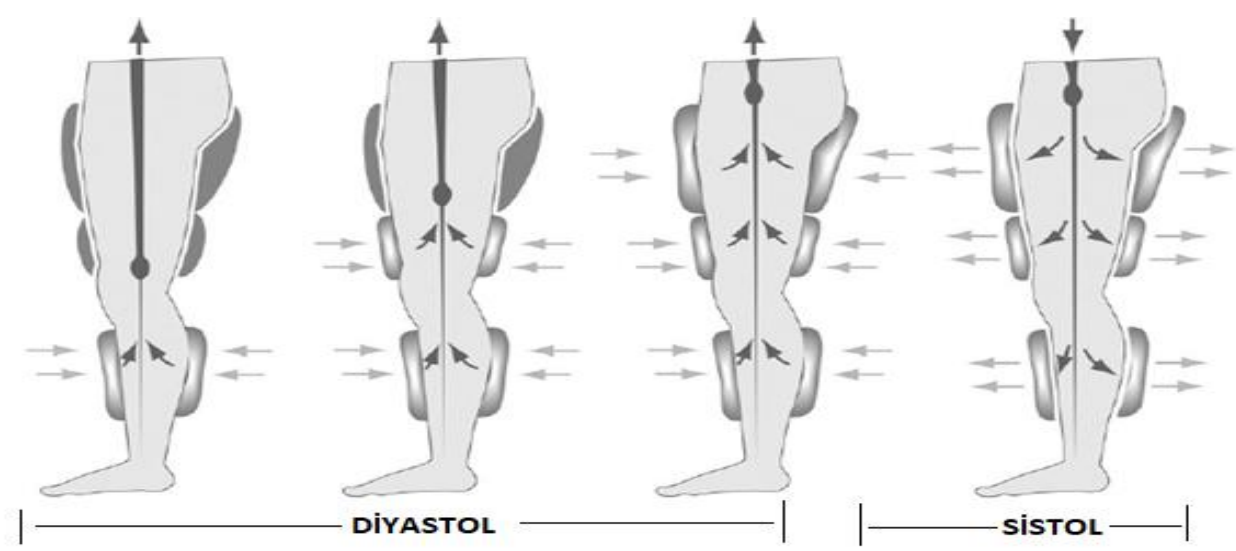


Bu çalışmada, EECP cihazının manşonlarının ST dalgasının başlangıcında sıkılıp sonunda ise gevşetilmesi için gerekli olan ST segmentasyon verisinin EKG sinyalleri üzerinden yapay sinir ağı ile elde edilmesi amaçlanmıştır. EECP, girişimsel bir yöntem olmadığı gibi farmakolojik bir tedavi biçimi de değildir. EECP tedavisinin çeşitli etki mekanizmaları olduğu düşünülmektedir. Bunlar; endotel fonksiyonda iyileşme, kolateral damar oluşumu, ventrikül fonksiyonunda gelişme, oksijen tüketiminde iyileşme, aterosklerozda gerileme ve periferde egzersize benzer antrenman etkisi olarak değerlendirilmektedir. Yaratttığ 1 hemodinamik etkiler intra aortik balon pompasının (Intra-aortic Balloon Pump - IABP) yarattığı etkilere benzer. Bununla birlikte IABP'den farklı olarak EECP venöz dönüşte de artış sağlamaktadır [5].

EECP’nin yol açtı̆ı̆ akut hemodinamik etkilerden yola çıkarak etki mekanizması üzerine şöyle bir hipotez geliştirilmiştir: EECP venöz dönüşü artırmakta, diyastolik takviye sağlamakta kardiyak debiyi ve koroner kan akışını yükseltmektedir. Tüm bu faktörler damar içi kayma geriliminin artmasına sebep olmaktadır. Kayma gerilimi ise bilindiği üzere anjiyojenesisi tetiklemekte ve vazoaktif maddeler yoluyla vasküler fonksiyonun iyileşmesine yol açmaktadır [6].

\section{Materyal ve Metot}

$\mathrm{Bu}$ çalışmada yapay sinir ağının eğitilmesi ve test edilmesinde PhysioNet'in sağlamış olduğu MIT-BIH Arrhytmia dataları kullanılmışıır. Datalar iki kanallı olup 47 adet yarım saatlik kayıtlardan oluşmaktadır. Bu kanallardan mML2, ve 45 adet sinyal kullanılmıştır. Çalışma, 20000000 adet veri ile çalıştırılarak sonuçları incelenmiştir. Üzerinde çalışılan bu veriler 11 bit çözünürlükte $10 \mathrm{mV}$ aralı̆̆ında olup saniyede 360 örnekleme şeklinde sayısallaştırılmıştır. Belirtilen sinyaller MATLAB R2016b programıyla işlenmiş̧ir.

Yapılan çalışmada üç temel adım izlenmiştir. Bu adımlardan ilki ön işleme ile ham sinyaldeki gürültü eliminasyonu ve baseline kaymalarını engellenmesidir. İkinci adım ile sinyalin P, Q, R, S, T noktalarının tespiti yapılmıştır. Son adımda ise ST noktaları elde edilmiş̧ir. (Şekil 3)

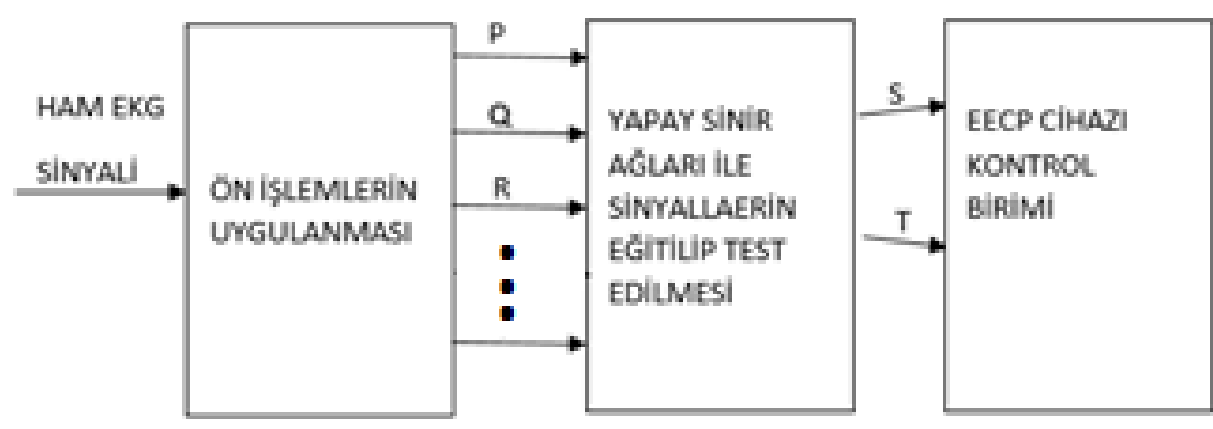

(a)
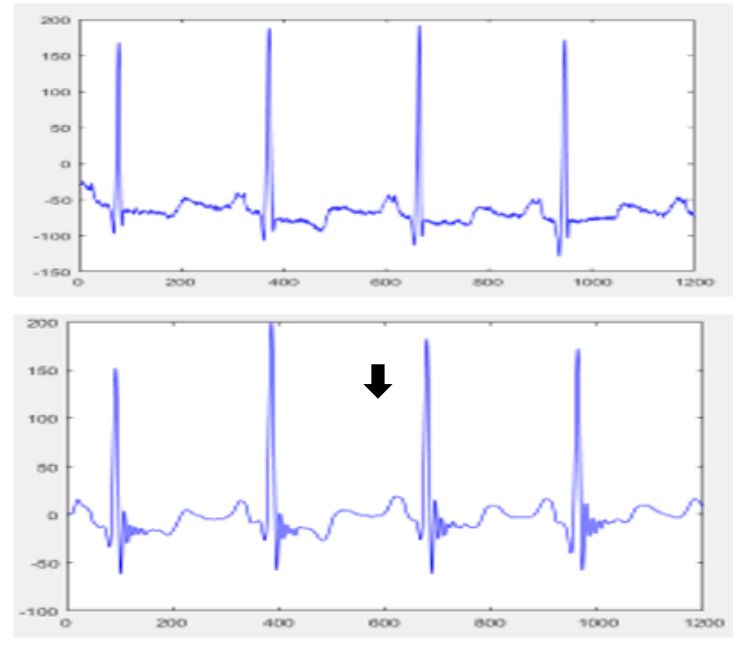


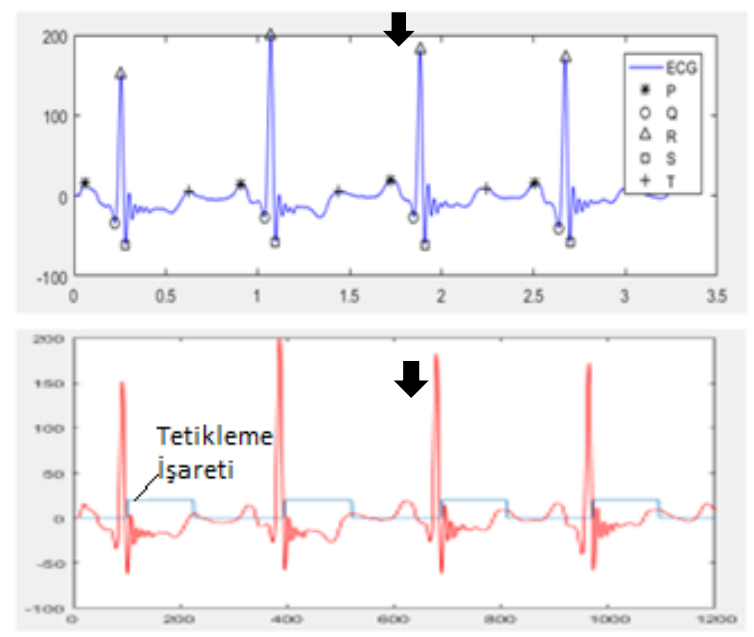

(b)

Şekil 3: Uygulanan metodun blok gösterimi(a) ve program üzerindeki uygulaması(b)

\section{1. Ön İșleme}

Ön işlemeye baseline'ın sıfira oturtulmasıyla başlanmıştır. İşaretin baseline'ı sıfira oturtmak için 9 seviyeli, 8 dizili Daubechies wavelet dönüşümü kullanılmıştır.

Baseline'1 sıfira oturtulan işaret, $60 \mathrm{~Hz}$ şebeke girişim gürültüsünden arındırılmak için çentik (notch) filtre ile diğer gürültülerden arındırılmak için ise band durduran ve alçak geçiren butterworth filtrelerinden geçirilmiştir.

\section{2. $P, Q, R, S$ ve $T$ Noktalarının Saptanması}

Yapılan çalışmada, üzerinde işlem yapılacak olan sinyalin gürültü eliminasyonu yapılıp ve baseline'ı da sıfira oturtulduktan sonra EKG işaretindeki noktalar tespit edilmiş̧ir. Bu noktaların tespitinde işaretin ön hazırlık aşamasında Pan ve Tompkins yöntemi kullanılmıştır [7]. Ancak tepe ve çukurların saptanmasında daha farklı bir yol izlenmiştir.

Ön işlemden geçirilen sinyal PQRST noktalarının belirlenebilmesi için yeni işlemlere tabi tutulmaktadır. Bu işlemlerden ilki, bir AC sinyal olan EKG sinyalinin içinde bulunan DC bileşenlerden arındırılması işlemidir. Genlik değerlerinin 0-1 arasında ifade edilmesi değerlendirme yaparken kolaylık sağlayacağı için sinyale normalizasyon işlemi uygulanmıştır. Normalize edilen sinyal, sırasıyla alçak ve yüksek geçiren filtrelerden geçirilmiştir. Daha sonra kareleme işlemi ve ardından da kayan pencere integratörü uygulanmıştır.

Yapılan bu işlemlerden sonra Pan ve Tompkins Algoritması'ndan farklı bir algoritma kullanılarak PQRST noktaları saptandı. Kullanılan bu algoritmada öncelikle işaretler her QRS dalgasına göre sol ve sağ bölgeler şeklinde sınıflandırılır. Ardından sol ve sağ olarak belirlenen bölgelerden sol bölge sonunun 60 birim daha solundaki yerde minimum işaret bulunarak P bölgesi olarak belirlenir. Sol ve sağ bölgeler arasında kalan maksimum tepecik de R tepesi olarak alınır. Sol kısım ile R tepeciği arasında kalan bölgedeki minimum nokta Q bölgesi olarak belirlenir. R tepeciğiyle sağ bölge arasında kalan minimum nokta S bölgesi olarak alınır. Geriye kalan $\mathrm{T}$ bölgesinin tespitinde ise sağ bölge başlangıcından 125 birim daha sağ kısım arasındaki maksimum tepecik de $\mathrm{T}$ noktası olarak belirlenir [8]. Belirlenen tüm bu bölgeler Şekil-2(b) de görüldüğü gibi farklı simgelerle işaretlenerek EECP için gerekli olan tetikleme işareti elde edilir.

\subsection{St Segmentasyonu}

Bu çalışmada ST segmentasyonu işlemi için yapay sinir ağlarından faydalanılmıştır. Yapay sinir ağları, biyolojik sinir ağlarından esinlenerek oluşturulan nümerik işlemler yardımıyla öğrenme işlemini gerçekleştirmektedir. Bu öğrenme işlemi ile ekonomi, tıp, mühendislik, endüstri gibi günlük hayatta karşıllaştığımız birbirinden farklı doğrusal olmayan problemlerin çözümünde kullanılmaktadır $[9,10,11]$.

Doğrusal olmayan sistem karakteristiklerini en az hata ile modelleyebilme potansiyeline sahip olmalarından dolayı yapay sinir ağları, fonksiyon yaklaştırma (eğri uydurma) alanında yaygın olarak kullanılmaktadır. Yapay sinir ağları kullanılarak ölçüm sisteminin kalibrasyon verisinden kolayca elde edilebilen ve öğrenme verisi olarak da adlandırılan giriş-çıkış veri çiftinden doğrudan olması da dolaylı olarak giriş çıkış ilişkisini ortaya koyan F fonksiyonu elde edilir [10].

Yapay sinir ağları, giriş katmanı, ara katman ve çıkış katmanı olmak üzere üç ana kısımdan meydana gelmektedir (Şekil 4). 


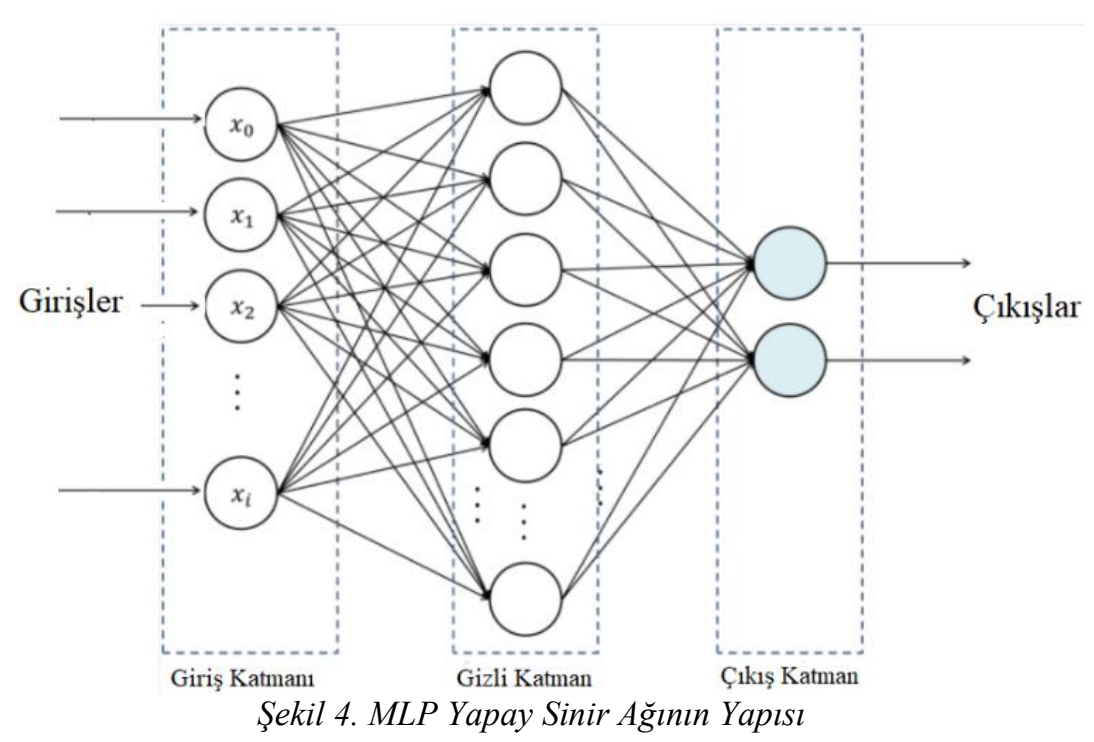

Yapay sinir ağlarını eğitmek için kullanılabilecek birçok eğitim algoritması mevcuttur. Bu algoritmalardan en çok kullanılanları aşağıda verilmiştir;

-Levenberg-Marquardt (trainlm)

-BFGS Quasi-Newton (trainbfg)

-Resilient Backpropagation (trainrp)

-Scaled Conjugate Gradient (trainscg)

-Conjugate Gradient with Powell/Beale Restarts (traincgb)

-Fletcher-Powell Conjugate Gradient (traincgf)

-Polak-Ribiére Conjugate Gradient (traincgp)

-One Step Secant (trainoss)

-Variable Learning Rate Backpropagation (traingdx)

$\mathrm{Bu}$ algoritmalar içerisinde en çok tercih edilen algoritma, fazla hafıza gerektirmesine rağmen hızlı olmasından dolayı geri beslemeli bir yapıya sahip olan Levenberg-Marquardt algoritmasıdır. Ağın eğitilmesi aşamasında kullanılan transfer fonksiyonları ise;

Gizli katman transfer fonksiyonları:

- Hyperbolic tangent sigmoid transfer function (tansig)

- Log-sigmoid transfer function (logsig)

- Elliot symmetric sigmoid transfer function (elliotsig)

Çıkış transfer fonksiyonları:

- Linear transfer function (purelin)

- Positive linear transfer function (poslin)

- Saturating linear transfer function (satlin)

- Symmetric saturating linear transfer function (satlins)

Oluşturulan algoritmada, doğrusal olmayan 10 girişli, 2 çıkışlı yapay sinir ağı k katlamalı çapraz doğrulama ile eğitilerek test edilmiştir. Oluşturulan yapay sinir ağında girişler $\mathrm{P}, \mathrm{Q}, \mathrm{R}$, ile bu noktalara yakın 7 farklı nokta, çıkışlar ise $\mathrm{S}$ ve T noktalarıdır. K katlamalı çapraz doğrulama, veri setini parçalara ayırma yöntemlerinden biridir. Bu yöntem, veriyi belirlenen bir k sayısına göre eşit parçalara böler, her bir parçanın hem eğitim hem de test için kullanılmasını sağlar, böylelikle dağılım ve parçalanmadan kaynaklanan sapma ve hataları minimuma indirir [12].

$\mathrm{Bu}$ çalışmada $\mathrm{k}$ katsayısını 2, 5, 10, 15 alarak, farklı gizli katman nöron sayıları, farklı eğitim algoritmaları ve transfer fonksiyonları kullanılarak en optimum sonucu veren yapı elde edilmeye çalışılmıştır. Hata analizinde test ortalama karesel hata (mse, mean squared error) değerleri irdelenmiştir.

K katlama değerleri irdelenirken optimum transfer fonksiyonlarını belirleyebilmek için parametrelerden sadece birini değiştirip diğer parametreleri sabit tutarak ağ eğitilmiş ve test mse değerleri kaydedilmiştir.

İkinci adımda elde edilen verilerle en uygun gizli katman nöron sayısı belirlenmiştir. Bu adımda hem logsig, purelin hem tansig, poslin hem de logsig, poslin şeklinde değişik kombinasyonlar kullanılarak en uygun gizli katman nöron sayıları için çalışma yapılmıştır. Elde edilen sonuçlar Tablo 1'de verilmiş̧ir.

Tablo 1: Optimum Transfer Fonksiyonları ve Değiş̧ik Nöron Sayllarının Eğitim Sonucu Hata Değerleri (Test mse) 
European Journal of Science and Technology

\begin{tabular}{|l|l|l|c|c|l|}
\hline $\begin{array}{c}\text { Gizli } \\
\text { Katman } \\
\text { Transfer } \\
\text { Fonk. }\end{array}$ & $\begin{array}{c}\text { Çıkış } \\
\text { Fonk. }\end{array}$ & $\begin{array}{c}\text { Ĕğitim } \\
\text { Algorit- } \\
\text { ması. }\end{array}$ & $\begin{array}{c}\text { Gizli } \\
\text { Katman } \\
\text { Nöron } \\
\text { Sayısı }\end{array}$ & $\begin{array}{c}\text { Fold } \\
\text { Sayısı }\end{array}$ & $\begin{array}{c}\text { Test Hatası } \\
\text { (MSE) }\end{array}$ \\
\hline logsig & purelin & trainlm & 10 & 5 & $2,96 \mathrm{E}-04$ \\
\hline logsig & purelin & trainlm & 15 & 5 & $2,52 \mathrm{E}-04$ \\
\hline logsig & purelin & trainlm & 20 & 5 & $2,32 \mathrm{E}-04$ \\
\hline logsig & purelin & trainlm & 25 & 5 & $2,31 \mathrm{E}-04$ \\
\hline tansig & poslin & trainlm & 10 & 5 & $3,20 \mathrm{E}-04$ \\
\hline tansig & poslin & trainlm & 15 & 5 & $2,99 \mathrm{E}-04$ \\
\hline tansig & poslin & trainlm & 20 & 5 & $2,55 \mathrm{E}-04$ \\
\hline tansig & poslin & Trainlm & 25 & 5 & $2,75 \mathrm{E}-04$ \\
\hline $\operatorname{logsig}$ & poslin & trainlm & 10 & 5 & $2,80 \mathrm{E}-04$ \\
\hline $\operatorname{logsig}$ & poslin & trainlm & 15 & 5 & $2,48 \mathrm{E}-04$ \\
\hline $\operatorname{logsig}$ & poslin & trainlm & 20 & 5 & $2,44 \mathrm{E}-04$ \\
\hline $\operatorname{logsig}$ & poslin & trainlm & 25 & 5 & $2,23 \mathrm{E}-04$ \\
\hline
\end{tabular}

Yapılan çalışma sonucunda optimum k katlama değeri 5, optimum transfer fonksiyonları da sırasıyla gizli katman transfer fonksiyonu için logsig, çıkış transfer fonksiyonu için ise poslin olarak belirlenmiştir. Gizli katmanda ise 25 nöron kullanılarak en küçük test hata değeri Tablo-1'den de görüleceği gibi $2,23 \times 10^{-4}$ olarak elde edilmiştir.

\section{Sonuç}

Sonuç olarak bu çalışmada, EKG işaretinin yapay sinir ağları kullanılarak ST segmentasyonu yapılmıştır. Elde edilen ST parçası, EECP sistemi kontrol biriminde tetikleme sinyali olarak kullanılarak manşonların zamanında sıkılıp gevşetilmesi işleminde kullanılabilecektir.

\section{Kaynakça}

[1] K. Surekha and B. Patil, "QRS Complex Compression Of ECG Signal Using Hybrid Transform," Bangalore, India, 20-21 Sept. 2013.

[2] O. Soran, "A New Treatment Modality in Heart Failure Enhanced External Counterpulsation (EECP)," Cardiology in Review, vol. 12, no. 1, pp. 15-20, 2004.

[3] A. Mokhlessi, M. Khalilzadeh, A. Moghimi and H. Soleiman, "EECP and Flow Rate of Coronary Arteries in Human Blood Circulation Model," in In 4th Kuala Lumpur International Conference on Biomedical Engineering , Berlin, 2008.

[4] S. YAVAŞ, "Güçlendirilmis Eksternal Kontrpulsasyon (EECP)," Türkiye Klinikleri Cardiovasc Sci, vol. 3, no. 19, pp. 165-173, 2007.

[5] O. SORAN, "Enhanced External Counterpulsation Therapy in Coronary Artery Disease Management," in Scientific Basis of Healthcare Angina, CRC Press, 2011, p. 174.

[6] M. A. SILVER, "The Role of Enhanced External Counterpulsation in Heart Failure Management," in Heart Failure , Device Management, Wiley Backwell, 2010, p. 151.

[7] P. JIAPU and W. J. TOMPKINS, "A Real Time QRS Detection Algoritm," IEEE transactions on biomedical engineering, pp. 230-236, march 1985.

[8] M. Bassiouni, E. El-Dahshan and W. K. e. al., "Intelligent hybrid approaches for human ECG signals identification," Springer, no. 12, pp. 941-949, 2018.

[9] E. Öztemel, Yapay Sinir Ağları, İstanbul: Papatya Yayıncılık Eğitim, 2012.

[10] İ. Dalkıran and K. Danışman, "E-Tipi Termal Çiftin Çıkışının Yapay Sinir Ağları ve Bulanık Sinir Ağları ile Doğrusallaştırılması," IEEE 14. Sinyal İşleme ve İletişim Uygulamaları Kurultayl, pp. 1-4, 2006.

[11] H. Erkaymaz, M. Özer and . İ. Orak, "Detection of directional eye movements based on the electrooculogram signals through an artificial neural network," Chaos, Solitons \& Fractals, pp. 225-229, May 2015.

[12] E. Şirin, "Bir Bakışta K-Fold Cross Validation," Veri Bilimi Okulu, 29 Ağustos 2017. [Online]. Available: https://www.veribilimiokulu.com/bir-bakista-k-fold-cross-validation/. [Accessed 29 Aralık 2019]. 